\title{
The Causal Effect of Switching to Second-line ART in Programmes without Access to Routine Viral Load Monitoring
}

\author{
Thomas Gsponera ${ }^{a}$ Maya Petersen ${ }^{b}$, Matthias Egger ${ }^{a, c}$, Sam Phiri ${ }^{d}$, Marloes H. Maathuis ${ }^{e}$, \\ Andrew Boulle ${ }^{c}$, Patrick Musondad ${ }^{f}, g$, Hannock Tweya ${ }^{d}$, Karin Peter ${ }^{e}$, Benjamin H. Chi ${ }^{g, h}$, \\ Olivia Keisera , and for leDEA Southern Africa
}

${ }^{a}$ Division of International and Environmental Health, Institute of Social and Preventive Medicine (ISPM), University of Bern, Switzerland bivision of Biostatistics, Berkeley School of Public Health, Berkeley, California, USA Infectious Diseases Epidemiology Unit, School of Public Health and Family Medicine, University of Cape Town, South Africa dLighthouse Centre, Kamuzu Central Hospital, Lilongwe, Malawi eSeminar for Statistics, ETH Zurich, Zurich, Switzerland fSchool of Medicine, Health Policy \& Practice, University of East Anglia, Norwich, UK ${ }^{9}$ Centre for Infectious Disease Research in Zambia (CIDRZ), Lusaka, Zambia hDepartment of Obstetrics and Gynecology, University of Alabama at Birmingham School of Medicine, Birmingham, USA.

\section{Abstract}

Objectives-We examined the effect of switching to second-line antiretroviral therapy (ART) on mortality in patients who experienced immunological failure in ART programmes without access to routine viral load monitoring in sub-Saharan Africa.

Design and setting-Collaborative analysis of two ART programmes in Lusaka, Zambia and Lilongwe, Malawi.

Methods-We included all adult patients experiencing immunological failure based on WHO criteria. We used Cox proportional hazards models weighted by the inverse probability of switching to compare mortality between patients who switched and patients who did not; and between patients who switched immediately and patients who switched later. Results are expressed as hazard ratios (HR) with $95 \%$ credible intervals (95\% CI).

Results-Among 2,411 patients with immunological failure 324 patients (13.4\%) switched to second-line ART during 3932person-years of follow-up. The median CD4 cell count at start of ART and failure was lower in patients who switched compared to patients who did not: 80 versus 155 cells $/ \mu \mathrm{L}(\mathrm{p}<0.001)$ and 77 versus 146 cells $/ \mu \mathrm{L}(\mathrm{p}<0.001)$, respectively.Adjusting for baseline and time-dependent confounders, mortality was lower among patients who switched compared to patients remaining on failing first-line ART: HR0.25 (95\%CI 0.09-0.72). Mortality was also lower among patients who remained on failing first-line ART for shorter periods: HR 0.70 (95\% CI 0.44-1.09) per 6 months shorter exposure.

Conclusion-In ART programmes switching patients to second-line regimens based on WHO immunological failure criteria appears to reduce mortality, with the greatest benefit in patients switching immediately after immunological failure is diagnosed.

Correspondence to Professor Matthias Egger, Institute of Social and Preventive Medicine (ISPM), University of Bern,

Finkenhubelweg 11, CH-3012 Bern, Switzerland. egger@ispm.unibe.ch. 


\section{Introduction}

The World Health Organization (WHO) estimates that over 6.6 million HIV-1 infected people were receiving antiretroviral therapy (ART) in low- and middle-income countries by the end of 2010[1]. As access to HIV treatment continues to expand, more people are experiencing treatment failure and use of second-line therapy is increasing [2]. In contrast to industrialized countries, where HIV-1 viral load is regularly measured, viral load monitoring is typically not available in the public-sector in resource-limited settings. At present, viral load monitoring is expensive and the necessary laboratory infrastructure is difficult to implement and maintain, particularly in rural areas.

In the absence of viral load monitoring, diagnosis of treatment failure relies on immunological (i.e. CD4 cell counts) and clinical criteria [3]. The ability of CD4 cell counts to predict virological failure is, however, limited: sensitivity and positive predictive value of the immunological WHO criteria for virological treatment failure have been shown to be poor [4]. Use of these criteria may therefore lead to unnecessary switching to second-line ART among patients with suppressed viral replication, and care givers appear to be reluctant to switch patients to second-line ART based on CD4 cell criteria: we previously found that in programmes relying on CD4 cell count monitoring patients switch later and at lower CD4 cell counts compared to programmes with access to viral load monitoring [5].

We know of no trials where patients meeting immunological failure criteria were randomized to remaining on first-line therapy or switching to second-line therapy, or to immediateversus delayed switching. In cohort studies confounding may distort associations between clinical outcomes and switching. Confounding by indication will bias results if switching is more likely in patients with lower CD4 cell counts at the time of immunologicalfailure, patients who are also more likely to die.Similarly, CD4 cell counts measured after failure may both affect the probability of subsequent switching and mortality; unlike standard confounders, however, time-updated CD4 cell counts may themselves be affected by prior switching decisions. Such time-dependent confounding can be seen as a special case of confounding by indication [6], which cannot be controlled for by standard multivariable adjustment.

We used marginal structural models and inverse probability weighting[7-9]to overcome this problem and estimate the effect of switching to second-line ART on all cause mortality in patients who experienced immunological failure in sub-Saharan Africa.

\section{Methods}

\section{The International epidemiological Databases to Evaluate AIDS}

The International epidemiological Databases to Evaluate AIDS (IeDEA) is a global research consortium with seven regional networks (including four networks in sub-Saharan Africa) to collect clinical and epidemiological data on HIV-infected people, and particularly patients on ART. The African networks of IeDEA have been described in detail elsewhere[10]. The Southern African region of IeDEA-SA includes ART programmes located in six countries (Botswana, Malawi, Republic of South Africa, Zambia, Mozambique, Zimbabwe). For the present study we included two well established ART programmes that rely on CD4 cell counts to detect treatment failure: the Ministry of Health (MoH)-Centre for Infectious Disease Research in Zambia (CIDRZ) programme in Lusaka, Zambia and the Lighthouse clinic at Kamuzu Central Hospital in Lilongwe, Malawi. The MoH-CIDRZ programme began to provide ART in 2004 across four sites in Lusaka. Since then, the programme has expanded to 68 facilities across four provinces, most of them government health centres and hospitals. 
In both programmes, clinical care is provided according to the national guidelines. During the study period individuals were eligible for treatment if (i) they were diagnosed with a stage IV condition according to World Health Organization (WHO) criteria; (ii) their CD4 cell count was below 200 cells/ $\mu \mathrm{L}$; or (iii) they had a stage III condition and their CD4 cell countwas between 200 and 350 cells $/ \mu \mathrm{L}$. Clinical and immunological monitoring occurred every 3 to 6 months. Viral load testing is available in Lusaka and Lilongwe and some patients in whom treatment failure is suspected are tested. In Lusaka viral load testing is generally performed if the clinical situation of the patient is perceived to be discordant with the CD4 cell count[11]. In Lilongwe some patients with immunological or clinical failure are tested. In both sites viral load testing is limited for reasons of cost and operational constraints.

\section{Inclusion criteria and definitions}

We included patients aged $>16$ years experiencing immunological failure after January 1 , 2004 based on any of the three WHO criteria: (i) CD4 cell counts staying persistently $<100$ cells $/ \mu \mathrm{L}$, (ii) a fall of CD4 cell counts below the baseline count, or (iii) a fall of $>50 \%$ from the peak value. Clinical stage was categorised into stage III or IV (advanced) and I or II (less advanced). Loss to follow-up was defined as not returning to the clinic for 12 months or longer.

\section{Statistical methods}

We split data into 3-month intervals (quarters). If no CD4 value was measured in a particular quarter, the last measured value was carried forward. Patients were followed up from the time of immunological failure to the date of database closure, death, or loss to follow up (i.e., 12 months after the last observed visit), whichever came first. We then estimated the causal effect of switching on mortality using two marginal structural models $[9,12]$. In the first model switching history was summarized ascurrent use of second-line therapy, while the second model contained in addition the time spent on first-line therapy since immunological failure. In both modelsthe following baseline covariates were used: CD4 cell count $(<50,50-99,100-199, \geq 200$ cells/ $\mu \mathrm{L})$, age $(<30,30-39,>40$ years $)$, sex, and clinical stage (advanced versus less advanced). The parameters of these marginal structural models were estimated using inverse probability of treatment weights (IPTW) [9].

In additional analyses we studied the causal effect of switching on mortality in patients who did versus those who did not have a viral load measurement between 6 months after starting ART and immunological failure, by including a term for the interaction between the availability of viral load measurements and switching in the outcome model, and including the availability of viral load measurement as predictor in the weight models. We investigated this issue further by estimating the causal effect of switching on mortality in patients from sites in the MoH-CIDRZ programme known to have no access to viral load measurements, and by including availability of a viral load measurement prior to switching as an additional time dependent confounder. Finally different models for the weights were investigated. The model is described in more detail in the Appendix.

All analyses were done in R-2.12.1 (R Project, University of Vienna, Austria) and Stata version 11 (Stata Corporation, College Station, TX, USA), and run independently at ETH Zurich and at ISPM Bern.Models were fit using Bayesian methodology with weakly informative prior distributions [13]. Results are reported as adjusted hazard ratios (HRs) with $95 \%$ credibility intervals $(\mathrm{CI})$ and conditional survival curves with $95 \%$ CIs. 


\section{Results}

Of 80,937 patients starting ART, 2,411 patients met eligibility criteria and experienced immunological failure in the period 2004 to 2009, including 2317 patients $(96.1 \%)$ from Zambia and 94 patients (3.9\%) from Malawi. A total of 324 patients (13.4\%) switched to a second-line ART regimen during 3932 person-years of follow-up, for anincidence of switching of 82.3 (95\% CI 73.7-91.6) per 1000 person-years. Seventy-six patients died, for an incidence of death of 19.2 (15.2-23.9) per 1000 person years and 177 patients were lost to follow-up for an incidence of 44.9 (38.6-51.89) per 1000 person-years. Incidence of loss to follow-up was lower among patients who did versus those who did not switch to a secondline regimen, $14.2(6.8-25.9)$ versus 50.5 (43.2-58.5)per 1000 person-years $(\mathrm{p}<0.001)$, respectively.

\section{Patient characteristics}

The age distribution at the start of ART was similar in patients who switched and patients who did not, with a median age of 26 years and 27 years, respectively (Table 1). In both groups there were slightly more women than men and close to three quarters of patients were in advanced clinical stages (WHO stage III or IV). The median CD4 cell count at the start of ART and at immunological failure was lower in patients who switched than in patients who did not: 80 versus 155 cells $/ \mu \mathrm{L}(\mathrm{p}<0.001)$ and 77 versus 146 cells $/ \mu \mathrm{L}$ $(\mathrm{p}<0.001)$, respectively. The most frequent first-line regimen consisted of Lamivudine (3TC), zidovudine (AZT) and nevirapine (NVP), followed by 3TC, stavudine (d4T) and NVP (Table 2). In both groups over $90 \%$ of patients started with one of these two regimens. Among patients who switched, the most frequent second-line regimen consisted of emitracibine (ETC), lopinavir (LPV) and tenofovir (TNV), followed by abacavir (ABC), didanosine (ddI) and LPV.

\section{Predictors of switching to second-line ART}

In the multivariate Cox model, patients with higher current CD4 counts were less likely to switch to second line therapy: the HR per 100 cells $/ \mu \mathrm{L}$ increase was $0.49(\mathrm{p}<0.001)$. Other covariates were not significantly associated with switching, although there was a trend towards a lower probability of switching among patients with baseline CD4 counts above 50 cells/ $\mu \mathrm{L}$ (Table 3).

\section{Effect of switching on mortality}

After adjusting for baseline and time-dependent confounders, the hazard of death among patients who had switched to second-line therapy immediately after failure was significantly lower than that ofpatientswho remained on an immunologically failingfirst-line regimen(HR $0.25,95 \%$ CI $0.09-0.72, \mathrm{p}=0.01$, upper panel of Figure 1 ). There was also a trend towards lower mortality among patients withshorter exposure to first-line therapy after immunological failure (HR 0.70 per 6 months shorter exposure, 95\% CI $0.44-1.09$, p=0.11, lower panel of Figure 1). These estimated causal hazard ratios were smaller than the crude (unadjusted) hazard ratios and the hazard ratios adjusted for baseline variables only. Figure 2 shows survival curves based on estimates from the weighted Cox models for women younger than 30 years in WHO clinical stage III/IV with baseline CD4 cell count between 100 and 199 cells $/ \mu \mathrm{L}$. Immediate switching resulted in an estimated $97.6 \%(95 \% \mathrm{CI}$ 92.3\%-99.2\%) survival at 5 years, compared to an estimated survival of $91.3 \%$ (95\% CI $84.7 \%-94.9 \%$ ) among women who remained on a first-line regimen. The estimated survival at 5 years among women switching 6 months, 12 months and 18 months after immunological failure was $96.6 \%$ (95\% CI 91.7\%-98.4\%), 95.6\% (95\% CI 90.9\%-97.7\%) and $94.7 \%$ (95\% CI 89.9\%-97.2\%), respectively. 


\section{Sensitivity analyses}

Overall 655 patients (27.2\%) had at least one viral load measured between 6 months after starting ART and immunological failure. Viral load measurements were more common among patients who switched (159 patients [49.1\%]) than among those who did not switch (496 patients [23.8\%], $\mathrm{p}<0.001)$. Detectable viral loads $(>400$ copies $/ \mathrm{ml})$ were measured in 137 patients $(86.2 \%)$ and 150 patients $(30.2 \%)$, respectively $(\mathrm{p}<0.001)$. The estimated HRs for switching in patients who did and did not have viral load measurementswere 0.13 (95\% CI $0.02-0.93$ ) and 0.40 (95\% CI 0.13-1.31), respectively ( $\mathrm{p}=0.30$ for difference), and the estimated HR including availability of a viral load test as a time dependent confounder was 0.44 (95\% CI 0.20- 0.99). When only considering patients from sites with no viral load measurements in the MoH-CIDRZ programme the HR was 0.46 (95\% CI 0.09-2.44). Finally, the causal effect of switching from the main analysis was insensitive to the different weight models, with HRs ranging between 0.20 and 0.30 .

\section{Discussion}

The accuracy of the WHO CD4 cell count criteria to detect virological failure is poor [4]. It is therefore not surprising that care givers are reluctant to switch patients to second-line therapy in ART programmes without access to viral load monitoring [5, 14, 15]. We aimed to clarify the causal effect of switching to second-line ART in such settings, using data from two large ART programmes in Zambia and Malawi. About 13\% of patients with documented immunological failure switched to second-line ART during a median of 1.7 years of follow-up. We found that mortality from all causes was reduced by about three quarters in patients who switched, compared to patients who did not, and that switching earlier reduced mortality compared to switching later.Our results also indicate that targeted viral load monitoring may further reduce mortality as it allows clinicians to selectively switch the patients who failed virologically and probably benefit most from second-line therapy.

The causal effect of switching to second-line therapy among immunologically failing patients should ideally be determined in a randomized controlled trial. However, it is unlikely that such a trial will ever be conducted: there must be ethical concerns about withholding an intervention that has a strong biological rationale, is standard in industrialized countries, and has been recommended by international bodies such as WHO. Observational data like those collected for this study may thus provide the best available evidence to inform clinical practice and public health decisions on whether and when to switch. Confounding by prognostic factors at baseline such as age, sex and clinical stage, andby time varying factors such as CD4 cell count may, however, distort the results from observational studies $[6,16]$. We used inverse probability of treatment weighting to adjust for confounding by time-updated CD4 cell counts. This approach avoids the problem that CD4 cell counts are intermediate on the causal pathway from switching to death: confounding is controlled by weights rather than by inclusion of CD4 cell count as a covariate in the statistical model.

The reduction in mortality associated with switching was substantial, but it may have been diluted by unknowingly switching patients who met the immunological criteria for failure but had undetectable viral load. In a previous study from the Lighthouse Clinic in Malawi, viral load was measured in 203 patients with immunological or clinical failure: virological failure was present in 54\% of patients, with high levels of viral resistance to NRTIs and NNRTIs[17, 18]. Positive predictive values were even lower in a collaborative analysis of 10 treatment programmes from Africa and Latin America [4]. In this study such dilution of the effect of switching will have been reduced by the targeted viral load measurement done in about half of patients who switched and about a quarter of patients who did not: viral load 
testing identifies virological failure and leads to switching in patients who benefit most. Indeed, in a sensitivity analysis we found that the reduction in mortality associated with switching was more pronounced in patients who had at least one viral load measurement compared to the patients without viral load measurements, although the difference failed to reach statistical significance. While confounding by viral load testing remains a concern, analyses restricted to sites without viral load testing and analyses in which availability of a prior viral load test was treated as a time dependent confounder both suggested that switching reduced mortality, although the former result failed to reach statistical significance. Our analyses were insufficiently powered to fully investigate these issues; we plan additional analyses as soon as more data on patients failing ART have become available.

Adherence is not consistently measured or reliablyrecorded in the participating cohorts and could therefore not be included in the analysis. Low levels of adherence are associated with poorer outcomes, both on first-line and second-line ART $[15,19]$ and patients perceived by their providers to be non-adherent might also be less likely to be switched, resulting in unmeasured confounding. In our study, adherence will generally have been lower among patients in whom ART failed, compared to patients on non-failing first-line ART.

Interestingly, the proportion of men in our study was higher than in a recent analysis of patients starting first-line ART in the two programmes [20], in line with the notion that adherence is lower in men than in women [21,22]. Men were, however, as likely to be switched as women, indicating that documented or perceived adherence was not playing a major role in decisions to switch or not to switch. Opportunistic infections and cancers were also not consistently recorded. Like adherence, opportunistic events could influence decisions; however, such confounding would be expected to lead to underestimation the benefit of switching. Of note, in an ART programme in Mozambique, Malawi and GuineaConakry only few patients met criteria for clinical failure without meeting immunological or virological failure criteria [23].

Less than $10 \%$ of patients were lost to follow-up after experiencing immunological failure. About twice as many patients are usually lost in the first year after starting ART [24] and about $40 \%$ of the patients lost to follow-up die in the following months[25]. Although follow-up was limited after starting second-line therapy, it is likely that in the present study mortality among patients lost was also high: all patients had failed immunologically and their CD4 cell count was therefore low by definition. We controlled for informative censoring to the extent possible by incorporating in the weights a factor reflecting the inverse of a subject's estimated probability of remaining under follow-upat that time[7]. Informative censoring remains a potential source of bias, however, particularly given the absence of death registries in Zambia and Malawi and the likelihood that some patients failed to return to the clinic because they had died[26].

We included two public-sector programmes involved in the scale-up of ART in Zambia and Malawi, with the Zambian programme contributing the vast majority (96.1\%) of patients. The generalizability of our results may therefore be questioned. However, the two programmes follow national guidelines for the public health approach to ART, which is recommended by WHO [27], and are typical for many other programmes in the region. Results should thus be applicable to many patients in a region heavily affected by HIV. We nevertheless acknowledge that the two sites included in this study will not be representative for all programmes in the region. For example, they are equipped with electronic medical record systems, have access to regular CD4 cell counts and are involved in an international collaboration of HIV cohorts[10]. 
To our knowledge this is the first study that examined the causal effect of switching to a second-line regimenamong patients with immunological failure in a low income setting using marginal structural models. A previous analysis of data from the United States of America [28] used marginal structural models to estimate the effect of switching to secondline therapy among patients with virological failure and found a hazard ratio of 0.81 in patients switching immediately compared to patients switching three months later. In a descriptive analysis of 11 ART programmes in sub-Saharan Africa, we found that mortality was higher in patients who switched compared to patients who remained on non-failing firstline therapy, and substantially higher in patients who remained on immunologically or virologically failing first-line ART[14]. These differences in mortality were not explained by differences in nadir CD4 cell count, age or sex, but time-dependent confounding was not considered[14].

In summary, our results suggest that in ART programmes in sub-Saharan Africa without access to routine viral load monitoring, patients meeting the WHO immunological failure criteria should be switched to a second-line regimen, and that delays in switching should be avoided. Our study also suggests that unnecessary switches might be avoided by targeted viral load; however, further research is needed to understand the barriers to switching patients who meet failure criteria, including, for example, the limited availability of secondline and third-line regimens, and the causal effect in patients with and without targeted viral load measurements. Finally, future studies should investigate at what CD4 cell countlevels patients should ideally be switched, similar to the question ofwhen ART should be started[29], and should examine long-term outcomes, including outcomes after second-line failure.

\section{Acknowledgments}

We thank all study participants and Marcel Zwahlen, Albert Mwango, Janne Estill, Gilles Wandeler and Lucy Campbell for helpful discussions and support. The study was supported by the National Institute of Allergy and Infectious Diseases (NIAID), Grant 5U01-AI069924-05 and a PROSPER fellowship to O.K.funded by the Swiss National Science Foundation (Grant 32333B_131629). The content is solely the responsibility of the authors and does not represent the official views of NIAID or the National Institutes of Health or the Swiss National Science Foundation.

\section{Appendix}

Confounding by indication will bias results if switching is more likely in patients with lower CD4 cell counts at the time of immunological failure; patients who are also more likely to die. Similarly, CD4 cell counts measured after failure may both affect the probability of subsequent switching and mortality; unlike standard confounders, however, time-updated CD4 cell counts may themselves be affected by prior switching decisions. Such timedependent confounding can be seen as a special case of confounding by indication [6], which cannot be controlled for by standard multivariable adjustment. These relationships can be visualized in a directed acyclic graph [30] (DAG), as shown in a simplified version in Figure A1.

To control for time dependent confounding we estimated the causal effect of switching on mortality using two Cox proportional hazards marginal structural models of the counterfactual hazard of death under alternative switching times, making the following assumptions: (1) no unmeasured confounding of the effect of switching on mortality, (2) a probability greater than zero of switching and not switching at each time point, regardless of covariate values and (3) correct specification of the treatment, censoring, lost to follow-up and outcome models. 
The parameters of these marginal structural models were estimated using inverse probability of treatment weights (IPTW) [9]. The inverse probability of treatment weights were stabilised and included additional censoring and loss to follow up components [8]. The treatment weight for a given time point was based on the inverse of a patient's estimated probability of having his or her observed switching history up to that time point, given his or her treatment and covariate history. Thus, for each quarter we estimated the probability of switching to second-line ART in that quarter among patients who had not already switched using Cox regression with predictors including all baseline covariates together with most recent CD4 cell count and the (lagged) CD4 cell count from the previous quarter. Most recent CD4 cell count refers to the last CD4 cell count before switching within a quarter. For each weight model three different structures were considered: (1) a simple linear model on the log-hazard scale including all predictors described above; (2) a linear model on the loghazard scale where nonlinear associations were incorporated using natural cubic splines and (3) the best fitting linear model on the log-hazard scale based on AIC forward/backward stepwise selection procedure.

We used generalised additive models to assess linearity of the association between CD4 cell count and the log-hazard of switching.

P-values correspond to tail probabilities of the coefficients' posterior distributions.

\section{References}

1. HIV treatment reaching 6.6 million people, but majority still in need. World Health Organization; Geneva: Jun 3. 2011

2. Keiser O, Orrell C, Egger M, Wood R, Brinkhof MW, Furrer H, et al. Public-health and individual approaches to antiretroviral therapy: township South Africa and Switzerland compared. PLoS.Med. 2008; 5:e148. [PubMed: 18613745]

3. World Health O. Antiretroviral therapy for HIV infection in adults and adolescents: recommendations for a public health approach. World Health Organization; Geneva: 2010. p. 1-359.2010 revision

4. Keiser O, MacPhail P, Boulle A, Wood R, Schechter M, Dabis F, et al. Accuracy of WHO CD4 cell count criteria for virological failure of antiretroviral therapy. Trop.Med.Int Health. 2009; 14:12201225. [PubMed: 19624478]

5. Keiser O, Tweya H, Boulle A, Braitstein P, Schecter M, Brinkhof MW, et al. Switching to secondline antiretroviral therapy in resource-limited settings: comparison of programmes with and without viral load monitoring. AIDS. 2009; 23:1867-1874. [PubMed: 19531928]

6. Sterne JA, Hernan MA, Ledergerber B, Tilling K, Weber R, Sendi P, et al. Long-term effectiveness of potent antiretroviral therapy in preventing AIDS and death: a prospective cohort study. Lancet. 2005; 366:378-384. [PubMed: 16054937]

7. Robins J, Hernan MA, Brumback B. Marginal structural models and causal inference in epidemiology. Epidemiology. 2000; 11:550-560. [PubMed: 10955408]

8. Hernan MA, Alonso A, Logan R, Grodstein F, Michels KB, Willett WC, et al. Observational studies analyzed like randomized experiments: an application to postmenopausal hormone therapy and coronary heart disease. Epidemiology. 2008; 19:766-779. [PubMed: 18854702]

9. Cole SR, Hernan MA. Constructing inverse probability weights for marginal structural models. Am.J Epidemiol. 2008; 168:656-664. [PubMed: 18682488]

10. Egger M, Ekouevi DK, Williams C, Lyamuya RE, Mukumbi H, Braitstein P, et al. Cohort Profile: The international epidemiological databases to evaluate AIDS (IeDEA) in sub-Saharan Africa. International Journal of Epidemiology. 2011

11. Goldman JD, Cantrell RA, Mulenga LB, Tambatamba BC, Reid SE, Levy JW, et al. Simple adherence assessments to predict virologic failure among HIV-infected adults with discordant immunologic and clinical responses to antiretroviral therapy. AIDS Res Hum Retroviruses. 2008; 24:1031-1035. [PubMed: 18724803] 
12. Hernan MA, Wolfe F, Tilling K, Choi H, Sterne JA. Controlling for time-dependent confounding using marginal structural modelsA1 - Fewell,Z. Stata Journal. 2004; 4:402-420.

13. Gelman A, Jakulin A, Pittau MG, Su YS. A weakly informative default prior distribution for logistic and other regression models. The Annals of Applied Statistics. 2008; 2:1360-1383.

14. Keiser O, Tweya H, Braitstein P, Dabis F, MacPhail P, Boulle A, et al. Mortality after failure of antiretroviral therapy in sub-Saharan Africa. Tropical medicine \& international health : TM \& IH. 2010; 15:251-258. [PubMed: 20003034]

15. Pujades-Rodriguez M, Balkan S, Arnould L, Brinkhof MA, Calmy A. Treatment failure and mortality factors in patients receiving second-line HIV therapy in resource-limited countries. JAMA : the journal of the American Medical Association. 2010; 304:303-312. [PubMed: 20639564]

16. Hernan MA, Brumback B, Robins J. Marginal structural models to estimate the causal effect of zidovudine on the survival of HIV-positive men. Epidemiology. 2000; 11:561-569. [PubMed: 10955409]

17. Hosseinipour MC, van Oosterhout JJ, Weigel R, Phiri S, Kamwendo D, Parkin N, et al. The public health approach to identify antiretroviral therapy failure: high-level nucleoside reverse transcriptase inhibitor resistance among Malawians failing first-line antiretroviral therapy. AIDS. 2009; 23:1127-1134. [PubMed: 19417582]

18. Hosseinipour MC, Kumwenda JJ, Weigel R, Brown LB, Mzinganjira D, Mhango B, et al. Secondline treatment in the Malawi antiretroviral programme: high early mortality, but good outcomes in survivors, despite extensive drug resistance at baseline. HIV Medicine. 2010; 11:510-518. [PubMed: 20345885]

19. Lima VD, Harrigan R, Bangsberg DR, Hogg RS, Gross R, Yip B, et al. The combined effect of modern highly active antiretroviral therapy regimens and adherence on mortality over time. Journal of Acquired Immune Deficiency Syndromes. 2009; 50:529-536. [PubMed: 19223785]

20. Keiser O, Chi BH, Gsponer T, Boulle A, Orrell C, Phiri S, et al. Outcomes of antiretroviral treatment in programmes with and without routine viral load monitoring in Southern Africa. AIDS. 2011 in press.

21. Hawkins C, Chalamilla G, Okuma J, Spiegelman D, Hertzmark E, Aris E, et al. Gender Differences in Antiretroviral Treatment Outcomes among HIV-infected Adults in Dar es Salaam, Tanzania. AIDS. 2011

22. Bastard M, Koita Fall MB, Laniece I, Taverne B, Desclaux A, Ecochard R, et al. Revisiting longterm adherence to HAART in Senegal using latent class analysis. Journal of Acquired Immune Deficiency Syndromes. 2011

23. Palombi L, Marazzi MC, Guidotti G, Germano P, Buonomo E, Scarcella P, et al. Incidence and Predictors of Death, Retention, and Switch to Second-Line Regimens in Antiretroviral-Treated Patients in Sub-Saharan African Sites with Comprehensive Monitoring Availability. Clin.Infect.Dis. 2008

24. Brinkhof MW, Dabis F, Myer L, Bangsberg DR, Boulle A, Nash D, et al. Early loss of HIVinfected patients on potent antiretroviral therapy programmes in lower-income countries. Bull World Health Organ. 2008; 86:559-567. [PubMed: 18670668]

25. Brinkhof MW, Pujades-Rodriguez M, Egger M. Mortality of patients lost to follow-up in antiretroviral treatment programmes in resource-limited settings: systematic review and metaanalysis. PLoS One. 2009; 4:e5790. [PubMed: 19495419]

26. Geng EH, Nash D, Kambugu A, Zhang Y, Braitstein P, Christopoulos KA, et al. Retention in care among HIV-infected patients in resource-limited settings: emerging insights and new directions. Current HIV/AIDS reports. 2010; 7:234-244. [PubMed: 20820972]

27. Gilks CF, Crowley S, Ekpini R, Gove S, Perriens J, Souteyrand Y, et al. The WHO public-health approach to antiretroviral treatment against HIV in resource-limited settings. Lancet. 2006; 368:505-510. [PubMed: 16890837]

28. Petersen ML, van der Laan MJ, Napravnik S, Eron JJ, Moore RD, Deeks SG. Long-term consequences of the delay between virologic failure of highly active antiretroviral therapy and regimen modification. AIDS. 2008; 22:2097-2106. [PubMed: 18832873] 
29. When to Initiate Combined Antiretroviral Therapy to Reduce Mortality and AIDS-Defining Illness in HIV-Infected Persons in Developed Countries: An Observational Study. Annals of Internal Medicine. 2011; 154:509-515. [PubMed: 21502648]

30. VanderWeele TJ, Hernan MA, Robins JM. Causal directed acyclic graphs and the direction of unmeasured confounding bias. Epidemiology. 2008; 19:720-728. [PubMed: 18633331] 

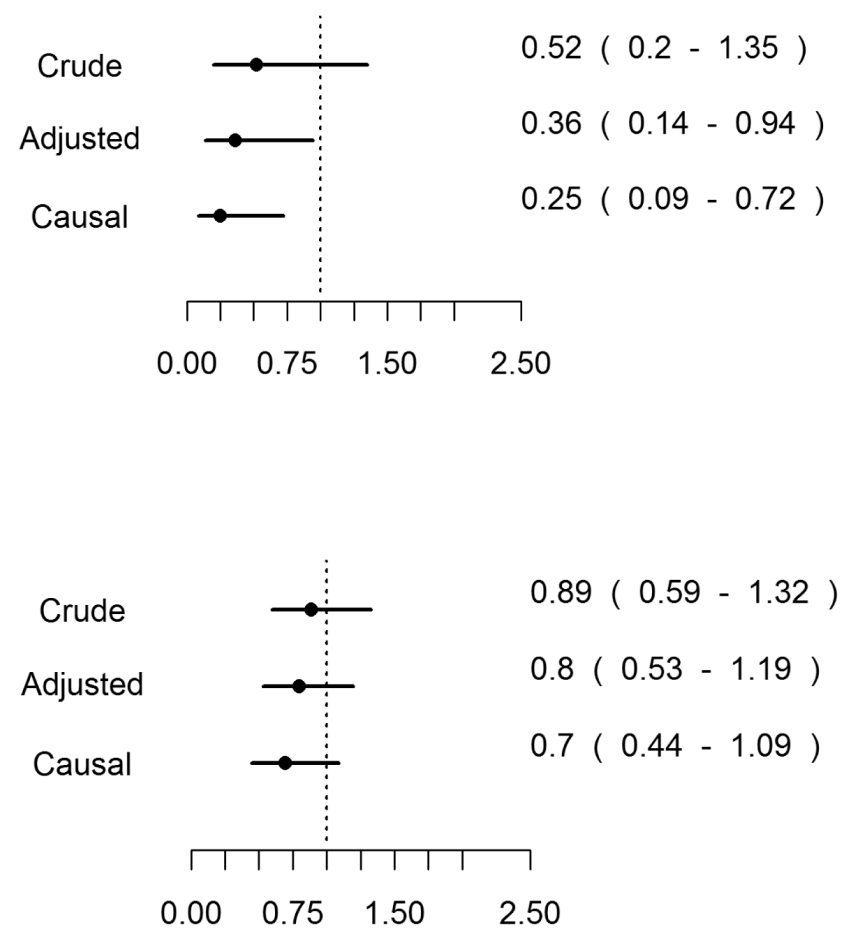

Figure 1. Estimated effect of switching to second-line therapy on all cause mortality among patients experiencing immunological failure, using unweighted(crude and adjusted) and weighted (causal) Cox models

Upper panel: HR for immediate switching compared to patients remaining on first-line regimen.

Lower panel: HR for each additionalsix month delay until switching.

Hazard ratios with $95 \%$ credibility intervals are shown. 


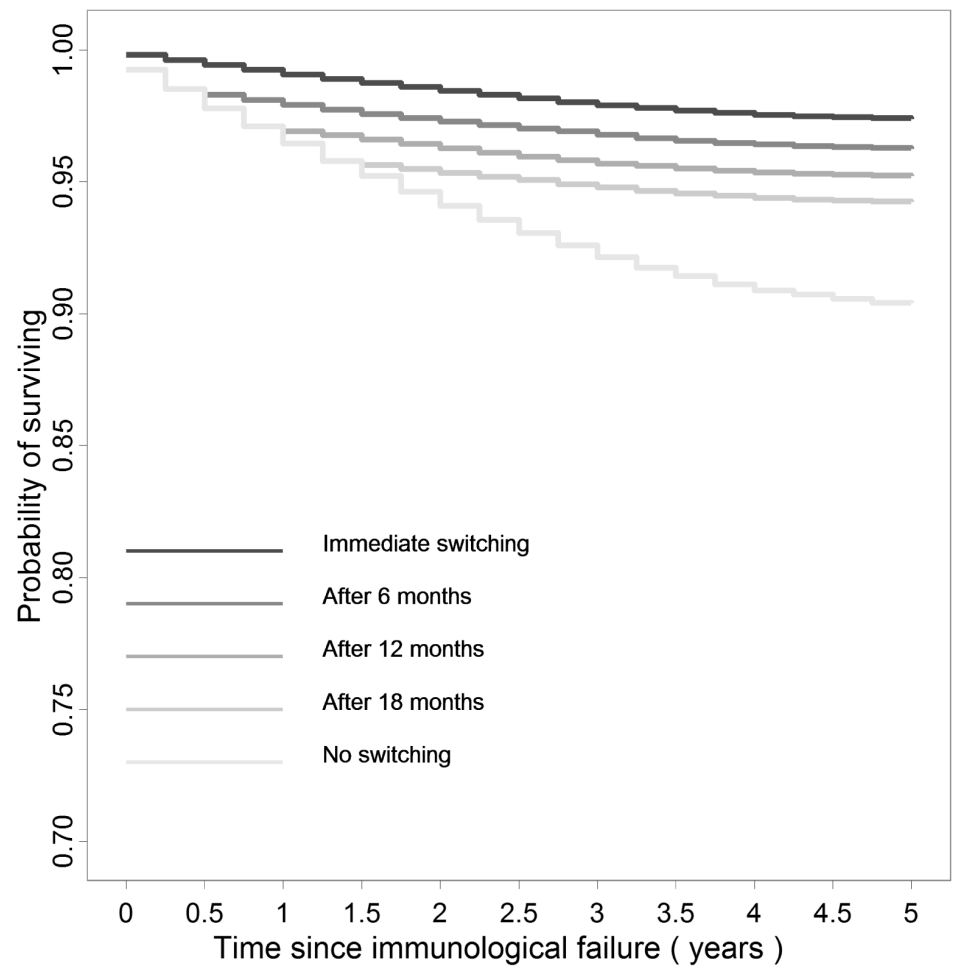

Figure 2. Predicted survival in women younger than 30 years in stage III/IV with a baseline CD4 cell count between 100 and 199 cells/ $\mu \mathrm{L}$ after immunological failure

Survival curves for immediate switching to second-line antiretroviral therapy, switching after 6,12 and 18 months and not switching are shown. 


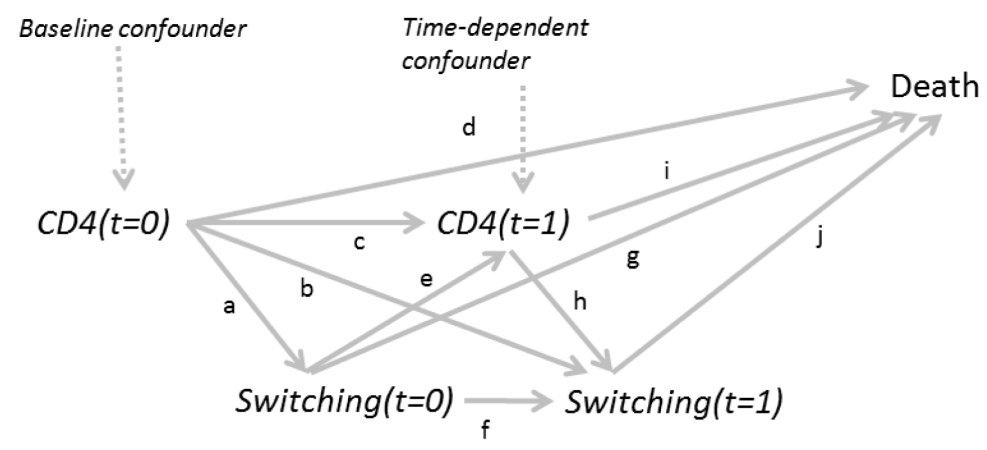

Figure A1. Directed acyclic graph (DAG) illustrating time-dependent confounding in a setting with 2 time points and omitting baseline and unmeasured confounders Arrows represent direct causal relationships between variables.

CD4 cell count at a given time point (e.g. $t=0)$ not only determines if therapy is switched to a second-line regimen at some time point (e.g. $\mathrm{t}=0$ or $\mathrm{t}=1$, arrows $\mathrm{a}$ and $\mathrm{b}$ ) but also influences CD4 cell count at a later time point (e.g. $\mathrm{t}=1$, arrow c) and mortality (arrow d). Switching at a given time point (e.g. $\mathrm{t}=0$ ) also influences CD4 cell count and switching at later time points (e.g. $\mathrm{t}=1$, arrows e and f) and mortality (arrow g). Similarly, CD4 cell count at time point $\mathrm{t}=1$ and switching at time point $\mathrm{t}=1$ influence mortality (arrows $\mathrm{h}$ and $\mathrm{i}$ ).

Finally, switching at time point $\mathrm{t}=1$ influences mortality (arrow $\mathrm{j}$ ). 


\section{Table 1}

Characteristics of patients failing first-line antiretroviral therapy based on immunological criteria at start of therapy and at failure, in patients who subsequently switched versus those who did not switch to second-line therapy

\begin{tabular}{|c|c|c|c|c|c|c|}
\hline & \multicolumn{3}{|c|}{ At start of ART } & \multicolumn{3}{|c|}{ At immunological failure } \\
\hline & $\begin{array}{l}\text { Switched } \\
(\mathrm{n}=324)\end{array}$ & $\begin{array}{l}\text { Did not switch } \\
(n=2,087)\end{array}$ & $\mathbf{P}$ & $\begin{array}{l}\text { Switched } \\
(\mathrm{n}=324)\end{array}$ & $\begin{array}{l}\text { Did not switch } \\
(\mathbf{n = 2 0 8 7})\end{array}$ & $\mathbf{P}$ \\
\hline Age (years) & & & 0.57 & & & 0.78 \\
\hline$<30$ & $215(66.4 \%)$ & $1368(65.5 \%)$ & & $179(55.3 \%)$ & $1157(55.5 \%)$ & \\
\hline $30-39$ & $93(28.7 \%)$ & $584(28.0 \%)$ & & $119(36.7 \%)$ & $740(35.4 \%)$ & \\
\hline$\geq 40$ & $16(4.9 \%)$ & $135(6.5 \%)$ & & $26(8.0 \%)$ & $190(9.1 \%)$ & \\
\hline Median (IQR) & $26(23-31)$ & $27(23-32)$ & & $29(25-33)$ & $29(25-34)$ & \\
\hline Sex & & & 0.76 & & & 0.76 \\
\hline Female & $169(52.2 \%)$ & $1107(53.1 \%)$ & & $169(52.2 \%)$ & $1107(53.1 \%)$ & \\
\hline Male & $155(47.8 \%)$ & $980(46.9 \%)$ & & $155(47.8 \%)$ & $980(46.9 \%)$ & \\
\hline $\begin{array}{l}\text { CD4 cell count } \\
(\text { cells } / \mu \mathrm{L}){ }^{*}\end{array}$ & & & $<0.001$ & & & $<0.001$ \\
\hline$<50$ & $99(35.1 \%)$ & $371(19.2 \%)$ & & $100(30.9 \%)$ & $225(10.8 \%)$ & \\
\hline $50-99$ & $61(21.6 \%)$ & $296(15.3 \%)$ & & $104(32.1 \%)$ & $481(23.1 \%)$ & \\
\hline 100-199 & $51(18.1 \%)$ & $539(27.9 \%)$ & & $87(26.8 \%)$ & $676(32.4 \%)$ & \\
\hline$\geq 200$ & $71(25.2 \%)$ & $726(37.6 \%)$ & & $33(10.2 \%)$ & $705(33.8 \%)$ & \\
\hline Median (IQR) & $80(32-204)$ & $155(64-279)$ & & $77(41-133)$ & $146(82-232)$ & \\
\hline WHO clinical stage & & & 0.73 & & & \\
\hline $\mathrm{I} / \mathrm{II}$ & $89(27.5 \%)$ & $555(26.6 \%)$ & & Not available & Not available & \\
\hline $\mathrm{III} / \mathrm{IV}$ & $235(72.5 \%)$ & $1532(73.4 \%)$ & & & & \\
\hline
\end{tabular}

* CD4 cell count at start of ART missing in 197 patients 


\section{Table 2}

Antiretroviral regimens in patients experiencing immunological failure, separately for those who switched and those who did not switch to second-line therapy

\begin{tabular}{lcc}
\hline & $\begin{array}{c}\text { Patients who switched } \\
(\mathbf{n = 3 2 4})\end{array}$ & $\begin{array}{c}\text { Patients who did not switch } \\
(\mathbf{n = 2 , 0 8 6})\end{array}$ \\
\hline First-line regimen & & \\
3TC AZT NVP & $172(53.1 \%)$ & $1043(50.0 \%)$ \\
3TC d4T NVP & $126(38.9 \%)$ & $879(42.1 \%)$ \\
3TC d4T EFV & $14(4.3 \%)$ & $75(3.6 \%)$ \\
3TC AZT EFV & $11(3.4 \%)$ & $86(4.1)$ \\
Other & $1(0.3 \%)$ & $3(0.2 \%)$ \\
Second-line regimen & & Not applicable \\
ETC LPV TNV & $194(59.9 \%)$ & \\
ABC DDI LPV & $65(20.1 \%)$ & \\
ATZ ETC LPV TNV & $15(4.6 \%)$ & \\
ATZ 3TC LPV/r TNV & $14(4.3 \%)$ & \\
Other & $36(11.1 \%)$ & \\
\hline
\end{tabular}

Numbers on regimen (\%) are shown.

$\mathrm{P}=0.65$ by chi-squared test for difference between first-line regimens. 
Table 3

Predictors of switching to second-line antiretroviral therapy after immunological failure

\begin{tabular}{lccl}
\hline & $\begin{array}{c}\text { Adjusted*Haza } \\
\text { rd ratio }\end{array}$ & 95\% CI & P \\
\hline $\begin{array}{l}\text { Current CD4 cell count } \\
\text { per 100 cells } / \mu \mathrm{L})\end{array}$ & 0.49 & $(0.36-0.67)$ & $<0.001$ \\
\hline $\begin{array}{l}\text { Lagged CD4 cell count } \\
\text { (per 100 cells } / \mu \mathrm{L})\end{array}$ & 0.98 & $(0.71-1.35)$ & 0.90 \\
\hline Age (years) & 1 & & \\
$<30$ & 1.08 & $(0.84-1.40)$ & \\
$30-39$ & 0.76 & $(0.46-1.27)$ & \\
$\geq 40$ & & & \\
$\quad$ Median (IQR) & & & \\
Sex & 1.11 & $(0.89-1.40)$ & 0.32 \\
Female & 1 & & \\
$\quad$ Male & & & \\
Baseline CD4 cell count \\
(cells/ $\mu \mathrm{L})$ \\
$<50$
\end{tabular}

